\title{
Some Alternatives for Robust Spectrum Estimation in Stationary Processes ${ }^{*}$
}

Fabio A. Fajardo ${ }^{* *}$

\begin{abstract}
This paper estimates the spectral density of stationary linear processes in the presence of additive outliers. The use of the robust periodogram, with different smoothing windows, proposed by Fajardo et al. (2009), is suggested. Empirical results reveal the robustness of the estimator in the presence of outliers. An application of the estimator is described for the estimation of the memory parameter in the IGP-DI series from August 1994 to April 2011.
\end{abstract}

Keywords: Robust Periodogram, Outliers, Robustness.

JEL Codes: C13, C15, C22.

${ }^{*}$ Submitted in October 2010. Revised in February 2012. The author thanks the comments and valuable suggestions made by Professor Valdério A. Reisen (UFES), by the Editor and by anonymous referees, who eventually contributed to improving this paper. The author takes full responsibility for any remaining errors. Finally, the author appreciates the financial support provided by CAPES/Brazil.

${ }^{* *}$ Department of Statistics. UFMG, Belo Horizonte, MG, Brazil.

Department of Statistics. UFES, Vitoria, ES, Brazil. E-mail: ffajardo@est.dout.ufmg.br 


\section{Introduction}

Time series analysis in the frequency domain is based on the spectral representation theorem. In stationary processes, parameter estimation can be obtained by minimizing the function

$$
\psi(\eta):=\sum_{j=1}^{\left\lfloor\frac{n}{2}\right\rfloor} \frac{I\left(\lambda_{j}\right)}{f\left(\lambda_{j} ; \eta\right)}
$$

where $\int_{-\pi}^{\pi} \log f(\lambda ; \eta) d \lambda=0, \lambda_{j}=\frac{2 \pi j}{n}$ are Fourier frequencies and $n$ is the sample size. The vector $\eta$ is the vector of unknown parameters, $I\left(\lambda_{j}\right)$ is referred to as periodogram and represents a spectrum estimator $f\left(\lambda_{j} ; \eta\right)$. The symbol $\lfloor\cdot\rfloor$ means the integer part function. However, time series in the presence of atypical observations or outliers (see Fox, 1972) have a significantly larger bias in the periodogram, affecting parameter estimation of the fitted models.

Fajardo et al. (2009) and Haldrup and Nielsen (2007) show that the increase in bias is caused by the presence of an additional term as a function of the magnitude of atypical data. The authors use Monte Carlo simulations to assess the effect of the presence of additive outliers on the estimation of the fractional differencing parameter in the $\operatorname{ARFIMA}(p, d, q)$ model. Estimators for $d$ based on the periodogram tend to underestimate the true parameter value in contaminated series, critically affecting series analyses.

To minimize the effect of outliers on parameter estimation, different methods have been proposed in the literature for robust spectrum estimation. For instance, Martin and Thomson (1982) suggest an algorithm based on a robust version of the Kalman filter, using generalized M estimators. Algorithm variants have been proposed by Tatum and Hurvich (1993) and Spangl and Dutter (2005). Those authors analyze the behavior of algorithms using Monte Carlo simulations, suggesting that the proposed methods could be robust alternatives to spectrum estimation. However, algorithms can have limitations in the case of stationary long-memory series Hampel et al. (1986, p. 422).

Fajardo et al. (2009) suggest a robust estimator for the spectrum of stationary processes contaminated by additive outliers. This estimator is known as robust smoothed periodogram and requires the application of the truncated window in order to reduce the contribution of final terms of autocovariance estimates and to make the estimator statistically consistent. The applications introduced by the authors suggest that the estimator is a robust alternative to the spectrum estimation of stationary processes.

The present paper assesses the behavior of the robust smoothed periodogram for different smoothing windows. With the use of Monte Carlo simulations, alternative windows improve the performance of the robust smoothed periodogram, minimizing bias and increasing the relative efficiency of the estimator. It is recommended 
that Parzen and Bartlett windows be used as they tend to yield the best values for accuracy measures in contaminated series. The robust periodogram is applied to the estimation of the fractional differencing parameter in $\operatorname{ARFIMA}(p, d, q)$ processes contaminated by additive outliers. The estimates for $d$ are obtained using a robust variant of the estimator proposed by Geweke and Porter-Hudak (1983), as suggested by Fajardo et al. (2009).

The paper is organized as follows. Section 2 provides the definitions of the lag windows applied to the robust periodogram. Section 3 defines the process contaminated by atypical data and analyzes the effects of contamination on spectrum estimators and on memory parameters in stationary processes. Section 4 describes the robust estimators for the autocovariance function and for the spectral density of stationary processes. The estimation results are shown in Section 5. Finally, Section 6 presents the major conclusions of the paper.

\section{Spectral Density Estimators}

Let $\left\{y_{t} ; t \in \mathbb{Z}\right\}$ be a stationary process with 0 mean and an autocovariance function given by

$$
R(h)=\int_{(-\pi, \pi]} e^{i h x} d F(x), \text { with } h \in \mathbb{Z},
$$

where $F(\cdot)$ is continuous on the right side, non-decreasing and limited on $[-\pi, \pi]$, $F(-\pi)=0$ and $F(\pi)=1$. The function $F(\lambda)=\int_{-\pi}^{\lambda} f(x) d x$ with $\lambda \in[-\pi, \pi]$ is designated as spectral distribution function and $f(\cdot)$ is the spectral density or the spectrum itself. If $\left\{y_{t} ; t \in \mathbb{Z}\right\}$ has a continuous spectrum, then

$$
f(\lambda)=\frac{1}{2 \pi} \sum_{h=-\infty}^{\infty} R(h) e^{-i h \lambda}, \text { with } \lambda \in[-\pi, \pi] .
$$

Let $\left\{y_{t} ; t=0,1, \ldots, n\right\}$ be a realization of the process $\left\{y_{t} ; t \in \mathbb{Z}\right\}$, a natural spectral estimator is defined by $\widehat{f}\left(\lambda_{j}\right)=\frac{1}{2 \pi} I\left(\lambda_{j}\right)$, such that

$$
I\left(\lambda_{j}\right)=\sum_{|h| \leq n} \widehat{R}(h) e^{-i h \lambda_{j}},
$$

where $\lambda_{j}=\frac{2 \pi j}{n}$, with $j=1,2, \ldots,\left\lfloor\frac{n}{2}\right\rfloor$ and $\widehat{R}(h)=\frac{1}{n} \sum_{i=1}^{n-|h|} y_{i+|h|} y_{i}$ is an estimator for $R(h)$. The periodogram function $I\left(\lambda_{j}\right)$ is an asymptotically unbiased estimator for the function $2 \pi f\left(\lambda_{j}\right)$, whose variance is given by $\operatorname{var}\left[I\left(\lambda_{j}\right)\right]=$ $(2 \pi)^{2} f^{2}\left(\lambda_{j}\right)+\mathcal{O}\left(n^{-1 / 2}\right)$, with $0<\lambda_{j}<\pi$. Note that, when $n \rightarrow \infty$, the estimator's variance does not tend towards zero, i.e., the periodogram is not a consistent estimator for the spectral density function (for details see, e.g., Brockwell and Davis, 2006). 
Smoothed versions of the estimator defined in Eq. 2 are suggested in order to make it statistically consistent. A smoothed estimator for spectral density is given by

$$
\widehat{f}_{s}\left(\lambda_{j}\right)=\frac{1}{2 \pi} \sum_{|k| \leq r} w_{n}(k) I\left(\lambda_{j+k}\right),
$$

where $r$ is known as bandwidth and satisfies $0<r<n$ with $r=o(n)$. The sequence $\left\{w_{n}(\cdot)\right\}$ satisfies the following conditions: $i$.) $w_{n}(k)=w_{n}(-k)$ and $w_{n}(k) \geq 0$ for every $k, i i.) \sum_{|k| \leq r} w_{n}(k)=1$, iii.) $\sum_{|k| \leq r} w_{n}^{2}(k) \rightarrow 0$ when $n \rightarrow \infty$. In particular, when $w_{n}(j)=\frac{1}{n} \sum_{|h| \leq r} \kappa\left(\frac{h}{r}\right) e^{-i h \lambda_{j}}$ one obtains approximately

$$
\widehat{f}_{s}\left(\lambda_{j}\right)=\frac{1}{2 \pi} \sum_{|h| \leq r} \kappa\left(\frac{h}{r}\right) \widehat{R}(h) e^{i h \lambda_{j}},
$$

where the function $\kappa(\cdot)$ is referred to as lag window and satisfies $\kappa(0)=1,|\kappa(x)| \leq$ 1 for every $x$ and $\kappa(x)=0$ if $|x|>1$ (for further details, see Anderson (1971) and Priestley (1981)). Some examples of lag windows ${ }^{1}$ :

i. truncated window $\kappa(x)=1$ if $|x| \leq r$, with $w_{n}(\theta)=D_{n}(\theta):=\frac{1}{2 \pi} \frac{\sin ((r+1 / 2) \theta)}{\sin (\theta / 2)}$. The asymptotic variance is given by $\frac{2 r}{n} f^{2}(\lambda)$. There is no closed formula for this type of window that allows assessing the estimator's bias Priestley (1981).

ii. Parzen window:

$$
\kappa(x)= \begin{cases}1-6 x^{2}+6|x|^{3}, & \text { if }|x| \leq 0.5 \\ 2(1-|x|)^{3}, & \text { if } 0.5<|x| \leq 1.0 \\ 0, & \text { if }|x|>1,\end{cases}
$$

with $w_{n}(\theta)=\frac{6}{\pi r^{3}} \frac{\sin ^{4}(r \theta / 4)}{\sin ^{4}(\theta / 2)}$. The asymptotic variance and bias are given, respectively, by $0.5393 \frac{r}{n} f^{2}(\lambda)$ and $-\frac{6}{r^{2}} f^{(2)}(\lambda)$, where $f^{(2)}(\lambda)=\frac{1}{2 \pi} \sum_{h=-\infty}^{\infty} h^{2}$ $R(h) e^{-i h \lambda}$.

iii. Tuckey-Hamming window: $\kappa(x)=0.54+0.46 \cos (\pi x),|x| \leq 1$, with $w_{n}(\theta)=$ $0.23 D_{n}(\theta-\pi / r)+0.54 D_{n}(\theta)+0.23 D_{n}(\theta+\pi / r)$. The asymptotic variance and bias are given, respectively, by $0.7948 \frac{r}{n} f^{2}(\lambda)$ and $-\frac{0.23 \pi^{2}}{r^{2}} f^{(2)}(\lambda)$.

\footnotetext{
${ }^{1}$ When selecting the smoothing window, a balance between bias and efficiency of the estimator should be maintained. In practice, statistical criteria are suggested for window selection (see, e.g., Beltrão and Bloomfield (1987) and Hurvich and Beltrão (1990)). The corresponding parameters for the windows defined above were selected according to the applications of Chen et al. (1994) and Reisen (1994) in the context of memory parameter estimation in fractional ARIMA processes.
} 
iv. Bartlett window: $\kappa(x)=1-|x|,|x| \leq 1$, with $w_{n}(\theta)=\frac{1}{2 \pi r} \frac{\sin ^{2}(r \theta / 2)}{\sin ^{2}(\theta / 2)}$. The asymptotic variance and bias are given, respectively, by $\frac{2 r}{3 n} f^{2}(\lambda)$ and $-\frac{1}{r} f^{(1)}(\lambda)$, where $f^{(1)}(\lambda)=\frac{1}{2 \pi} \sum_{h=-\infty}^{\infty}|h| R(h) e^{-i \lambda h}$.

\section{Contaminated Processes}

Following Martin and Yohai (1986), the contaminated process $\left\{z_{t} ; t=1,2, \ldots\right.$, $n\}$ is represented by the model

$$
z_{t}=y_{t}+v_{t}^{*}
$$

where $\left\{v_{t}^{*}\right\}$ is a sequence of random variables with distribution $F_{v^{*}}=(1-\gamma) \delta_{0}+$ $\gamma \mathcal{N}\left(0, \sigma_{v^{*}}^{2}\right)$, where $\delta_{0}$ represents the distribution with mass concentrated in value 0 and $\gamma \in[0,1]$. The variables $y_{t}$ and $v_{t}^{*}$ are independent for each value of $t$.

Fajardo et al. (2009) show that the periodogram of the process contaminated by atypical additive data is given by

$$
I_{z}(\lambda)=I_{y}(\lambda)+\Delta\left(\sigma_{v^{*}}^{2}\right), \quad \lambda \in(0, \pi)
$$

where $I_{y}\left(\lambda_{j}\right)$ is defined as in Eq. 2. An equivalent result is obtained for the estimator defined in Eq. 3. In the contaminated case, the statistical criteria for the selection of an optimal window for the smoothed estimator, defined in Eq. 3, lose their validity, and it is recommended that these criteria not be used when the presence of atypical data is suspected.

The term $\Delta\left(\sigma_{v^{*}}^{2}\right)$ increases the bias of spectrum estimators, affecting the parameter estimations obtained from the periodogram function. For example, in the estimation of the fractional differencing parameter in stationary $\operatorname{ARFIMA}(0, d, 0)$ processes in the presence of additive outliers, the usual estimators for the fractional differencing parameter have a negative bias, suggesting that the periodogrambased estimators tend to underestimate the true value of parameter $d$ in contaminated series. In particular, for the LP estimator, proposed by Geweke and Porter-Hudak (1983), and given by

$$
\widehat{d}_{y}=-0.5 s_{x}^{-1} \sum_{i=1}^{m} a_{i} \ln \widehat{f}\left(\lambda_{i}\right),
$$

where $a_{i}=x_{i}-\bar{x}$ with $x_{i}=\ln \left\{2 \sin \left(\frac{\lambda_{i}}{2}\right)\right\}^{2}, \bar{x}=m^{-1} \sum_{i=1}^{m} x_{i}, s_{x}=\sum_{i=1}^{m} a_{i}^{2}$, $m=n^{\alpha}, 0<\alpha<1$ and the function $\widehat{f}\left(\lambda_{i}\right)$ is defined in Eq. 2; one gets the following result:

Let $\left\{z_{t} ; t=1,2, \ldots, n\right\}$ be a contaminated process with additive outliers, with representation given by Eq. 4 , with $\left\{y_{t} ; t=1,2, \ldots, n\right\}$ being a stationary process, such that

$$
y_{t}=(1-B)^{-d} u_{t}, \quad d \in(0,0.5)
$$


where $\left\{u_{t} ; t=1,2, \ldots, n\right\}$ is an ARMA process. The filter of the fractional differentiation $(1-B)^{d}$ is defined by the binomial expansion $(1-B)^{d}=\sum_{j=0}^{\infty} \pi_{j} B^{j}$, where $\pi_{j}=\frac{\Gamma(j-d)}{\Gamma(j+1) \Gamma(-d)}$ with $\Gamma(\cdot)$ being the Gamma function. The bias of the LP estimator in contaminated processes is given by

$$
\mathbb{E}\left[\widehat{d}_{z}-d\right]=-\frac{\gamma(1-\gamma) \sigma_{v^{*}}^{2}}{2 \pi f_{u}(\lambda)}\left(2 \pi n^{-1} m\right)^{2 d}+O\left(m^{-1} \ln ^{3} m\right)+o\left(\left(n^{-1} m\right)^{2 d}\right),
$$

where $0<f_{u}(\lambda)<\infty$ is a continuous function at $\lambda \in[-\pi, \pi], f_{u}^{\prime}(0)=0$ and $\left|f_{u}^{\prime \prime}(\lambda)\right|<\infty$ for $\lambda$ in a neighborhood of 0 ; and the variance of the estimator is given by

$$
\operatorname{var}\left(\widehat{d}_{z}\right)=\frac{\pi^{2}}{24 m}+O\left(\left(n^{-1} m\right)^{4 d} \ln ^{2} m\right)+o\left(m^{-1}\right),
$$

when $n \rightarrow \infty, m \rightarrow \infty$ and $\left(n^{-1} m\right)^{2 d} \ln ^{2} m \rightarrow 0$.

Theorem 1 results indicate that the estimator proposed by Geweke and PorterHudak (1983) is negatively biased as a consequence of the magnitude of atypical data, suggesting that the LP estimator tends to underestimate the true value of parameter $d$ in contaminated series. Results suggest that the estimator's variance does not change significantly in the presence of outliers.

The presence of atypical data produces significant effects on memory parameter estimations, even when the percentage of outliers is small. The number of $m$ frequencies may cause changes in the estimator's bias. In uncontaminated series, Hurvich et al. (1998) calculate an optimal value for $m$ of order $n^{4 / 5}$. In an attempt to play down the influence of atypical data on the estimation of parameter $d$, Haldrup and Nielsen (2007) suggest the use of a smaller number of frequencies in the calculation of the LP estimator; however, there are no theoretical results that allow obtaining an optimal value for $m$ when the series are contaminated.

The generalization of Theorem 1 results when the spectral estimator is given by Eq. 3 can be obtained in an equivalent fashion by applying Theorem 9.4.2., p. 541 in Anderson (1971) and Theorem 1 result, p. 689 in Deo and Hurvich (2001). In this case, the estimator's bias for parameter $d$ is affected by the bandwidth value of the smoothed periodogram. An alternative to choose the value of $r$ is to use Monte Carlo simulations using a criterion that allows minimizing the accuracy of the smoothed estimator with respect to the spectral density function of the process, but the selection of an optimal bandwidth value does not correct the bias caused by the term $\Delta\left(\sigma_{v^{*}}^{2}\right)$ in Eq. 5 . To minimize the effect caused by outliers on spectral density estimators, it is suggested that robust estimators be used for the spectrum of stationary processes, as outlined below.

\section{Robust Estimators}

Using the expression shown in Eq. 3, a robust estimator is suggested for the spectral density of stationary processes, defined by 


$$
\widehat{f}_{r o b}\left(\lambda_{j}\right)=\frac{1}{2 \pi} \sum_{|h| \leq r} \kappa\left(\frac{h}{r}\right) \widetilde{R}(h) e^{i h \lambda_{j}},
$$

where $\lambda_{j}=\frac{2 \pi j}{n}$ with $j=1,2, \ldots,\left\lfloor\frac{n}{2}\right\rfloor$ and $n$ is the number of observations. The function $\widetilde{R}(\cdot)$ stands for a robust estimator for autocovariance, as suggested by Ma and Genton (2000), and is given by

$$
\widetilde{R}(h)=\frac{1}{4}\left[Q_{n-h}^{2}(\mathbf{u}+\mathbf{v})-Q_{n-h}^{2}(\mathbf{u}-\mathbf{v})\right],
$$

where $\mathbf{u}$ and $\mathbf{v}$ are vectors belonging to the first and last $n-h$ observations, respectively. The estimator $Q_{n}(\cdot)$ calculates the $k$-th order statistic of $\left(\begin{array}{l}n \\ 2\end{array}\right)$ distances $\left\{\left|z_{i}-z_{j}\right|, i<j\right\}$, i.e., $Q_{n}(z)=c_{\Phi} \cdot\left\{\left|z_{i}-z_{j}\right| ; i<j\right\}_{(k)}$, where $z=\left(z_{1}, z_{2}, \ldots, z_{n}\right)^{\prime}$ is the vector of data, $c_{\Phi}$ is a constant that guarantees consistency $\left(c_{\Phi}=2.2191\right.$ for the normal distribution) and $k=\left\lfloor\frac{\left(\begin{array}{l}n \\ 2\end{array}\right)+2}{4}\right\rfloor+1$.

Fajardo et al. (2009) suggest the use of the estimator defined in Eq. 7, smoothed with the truncated window in the estimation of long-memory stationary process parameters in the presence of atypical data. The authors analyze the empirical behavior of the estimator using Monte Carlo simulations, suggesting that the robust periodogram smoothed with the truncated window could be a robust alternative to the spectrum estimation of contaminated stationary processes.

The statistical properties of the estimator $\widetilde{R}(\cdot)$ are tested by Lévy-Leduc et al. (2011b) and Ma and Genton (2000). The authors show that, for $0 \leq h<n$, the function $\widetilde{R}(h)$ satisfies the central limit theorem, i.e.,

$$
n^{1 / 2}(\widetilde{R}(h)-R(h)) \stackrel{\mathcal{D}}{\longrightarrow} \mathcal{N}\left(0, \sigma_{h}^{2}\right)
$$

where $\sigma_{h}^{2}=\mathbb{E}\left[\tau^{2}\left(z_{1}, z_{1+h}\right)\right]+2 \sum_{j \geq 1} \mathbb{E}\left[\tau\left(z_{1}, z_{1+h}\right) \tau\left(z_{j+1}, z_{j+1+h}\right)\right]$. The function $\tau(\cdot)$ is such that

$$
\tau(x, y)=a^{+} \mathrm{FI}\left(\frac{x+y}{\sqrt{2 a^{+}}}, Q, \Phi\right)-a^{-} \mathrm{FI}\left(\frac{x-y}{\sqrt{2 a^{-}}}, Q, \Phi\right),
$$

where $\quad a^{+}=R(0)+R(h), \quad a^{-}=R(0)-R(h) \quad$ and $\quad F I(x, Q, \Phi)=c_{\Phi}$ $\frac{\frac{1}{4}-\Phi\left(x+c_{\Phi}^{-1}\right)+\Phi\left(x-c_{\Phi}^{-1}\right)}{\int \phi(x) \phi\left(x+c_{\Phi}^{-1}\right) d x}$. The function $Q:=Q(\Phi)$ represents a statistical functional associated with $Q_{n}(\cdot)$ while the functions $\Phi$ and $\phi$ denote the cumulative distribution and probability distribution functions of a standard Gaussian random variable, respectively. The expression in Eq. 9 refers to the influence function for a statistical functional $R(\cdot)$ associated with $\widetilde{R}(\cdot)$ (for details, see Ma and Genton, 2000, p. 675).

In series contaminated by atypical data, Ledolter (1989) shows that the effect of outliers on parameter estimations differs from their position along the series, 
i.e., the effect of an outlier is different if it is located at the beginning, middle, or end of the series. To deal with that, Ma and Genton (2000) suggest defining a temporal breakdown point ${ }^{2}$. The definition takes into consideration the time of occurrence of the atypical data along the time series, defined as

$$
\begin{gathered}
\gamma_{\text {temp }}^{*}\left(\widehat{R}_{\eta}(h)\right)=\max \left\{\frac{m}{n}: \sup _{\mathbb{I}_{m}} \sup _{\widetilde{\eta}} S_{n-h}(\widetilde{\mathbf{u}}+\widetilde{\mathbf{v}})<\infty, \inf _{\mathbb{I}_{m}} \inf _{\widetilde{\eta}} S_{n-h}(\widetilde{\mathbf{u}}+\widetilde{\mathbf{v}})>0,\right. \\
\left.\sup _{\mathbb{I}_{m}} \sup _{\widetilde{\eta}} S_{n-h}(\widetilde{\mathbf{u}}-\widetilde{\mathbf{v}})<\infty \text { and } \inf _{\mathbb{I}_{m}} \inf _{\widetilde{\eta}} S_{n-h}(\widetilde{\mathbf{u}}-\widetilde{\mathbf{v}})>0\right\},
\end{gathered}
$$

where $\widetilde{\eta}$ is an $n$-vector with $m$ values replaced with arbitrary values, $\widetilde{\mathbf{u}}$ and $\widetilde{\mathbf{v}}$ are obtained from $\widetilde{\eta}$ as in Eq. 8, $\mathbb{I}_{m}$ is a set of $m$ indices such that $\mathbb{I}_{m} \subset\{1,2, \ldots, n\}$ and $S_{n}(\cdot)$ is a scale estimator. $\widehat{R}_{\eta}(\cdot)$ is an estimator of function $R(\cdot)$. The function $\gamma_{\text {temp }}^{*}(\cdot)$ is known as temporal breakdown point ${ }^{3}$ and for estimator $\widetilde{R}(\cdot)$ one has that $\gamma_{\text {temp }}^{*}(\widetilde{R}(h)) \leq 0.25$, for $0 \leq h<n$.

Following the definition in Eq. 6, a robust estimator for $d$ can be defined by

$$
d_{L P R}=-0.5 s_{x}^{-1} \sum_{i=1}^{m} a_{i} \ln \widehat{f}_{r o b}\left(\lambda_{i}\right)
$$

where $\widehat{f}_{\text {rob }}\left(\lambda_{i}\right)$ is given in Eq. 7. The use of lag windows in estimator $\widehat{f}_{\text {rob }}\left(\lambda_{i}\right)$ allows reducing the contribution of irregular terms of the robust autocovariance function, given the calculation of the quantiles of the differences in function $Q_{n}(\cdot)$ (see Fajardo et al. (2009)).

\section{Empirical Studies}

In what follows, we present the results of Monte Carlo simulations used to assess the behavior of the estimator defined in Eq. 7 in series contaminated by additive outliers. Accuracy measures were calculated for the smoothed periodograms

\footnotetext{
${ }^{2}$ Rousseeuw and Croux (1993) showed that the breakdown point (BP) of the estimator $Q_{n}(\cdot)$ is $50 \%$, i.e., $50 \%$ of the observations can be replaced with atypical data and that the statistical properties of the estimator are not affected significantly. In the case of the autocovariance estimator, Ma and Genton (2000) demonstrate that the temporal breakdown point of $\widetilde{R}(\cdot)$ is smaller than or equal to $25 \%$.

${ }^{3}$ In the analysis of time series contaminated by atypical data, the optimal selection of parameter $r$ is still an open problem. The statistical criteria suggested in the literature lose their practical meaning when series have any type of contamination. The empirical results in Fajardo et al. (2009) show that it is necessary to have a balance between the temporal breakdown point and the bias of the estimator for bandwidth selection. The authors suggest applying the estimator in Eq. 7, using the truncated window with $r=\min \left\{h^{\prime}, G(n)\right\}$, where $G(n)=n^{\beta}, \beta \in(0,1)$ and $h^{\prime}=\min \left\{0<h<n: \varepsilon_{n}^{t e m p}\left(\widehat{R}_{Q}(h)\right) \leq \frac{m}{n}\right\}-1$. The value of $\beta$ can be obtained by Monte Carlo simulations using statistical selection criteria.
} 
as far as the theoretical spectrum of uncontaminated processes are concerned. The asymptotic relative efficiency (ARE) of the robust periodogram is obtained from the quotient between the variances of the estimator defined in Eq. 2 and the smoothed periodogram, obtained from Eq. 7 using Parzen, Tuckey-Hamming $(\mathrm{TH})$, Bartlett, and truncated windows. The windows are applied with different values of $r=n^{\beta}$, where $\beta=0.7,0.8,0.9$ and $n=300$. The contaminated series were obtained from the model in Eq. 4, with $\gamma=0,0.05,0.1,0.25$ and $\sigma_{v^{*}}=10$.

Additionally, an application is performed for the general price index (IGPDI) data from August 1994 to April 2011. Assuming that the IGP-DI series follows an ARFIMA process, the memory parameter is estimated by the estimators suggested in Eq. 6, with the periodogram defined in Eq. 2 and the estimator defined in Eq. 10 using Parzen, TH, Bartlett, and truncated windows. The simulations were conducted in $0 \mathrm{x}$ matrix programming language, available from http://www.doornik.com.

\subsection{Accuracy measures}

The results displayed in Table 1 show the accuracy measures for the smoothed estimator when Parzen, TH, Bartlett, and truncated windows were used. The table provides the root mean-squared error (RMSE), the mean absolute error (MAE) and the mean absolute percentage error (MAPE) of estimators with respect to the theoretical spectrum of the process. The boldface values represent the lowest measures for different periodograms. The process under analysis follows an $\operatorname{ARMA}(1,1)$ representation with $\phi=0.5$ and $\theta=0.3$ with different contamination levels. Note that the smoothed estimator is more accurate for $\beta=0.7$, when the Bartlett window is applied to contaminated series. The periodogram smoothed with Parzen window yields a better performance for uncontaminated series, whereas the truncated window yields the highest accuracy measures, suggesting that Parzen, TH and Bartlett windows can be regarded as reasonable alternatives to improving the spectral performance of ARMA processes in the presence of atypical observations.

Figure 1 shows the behavior of the smoothed periodogram in terms of the theoretical spectrum of an $\operatorname{ARMA}(1,1)$ process with $\phi=0.5$ and $\theta=0.3$, for different contamination levels and bandwidth calculated for $\beta=0.7$. The usual estimator of spectrum $\widehat{f}\left(\lambda_{j}\right)$ with $0<\lambda_{j}<\pi$, defined as a function of Eq. 2 , is denoted by $L S$.

The value $\beta=0.8$ can be considered an alternative value to the calculation of the bandwidth of the smoothed periodogram, given that the performance of the smoothed estimator does not demonstrate a significant increase in accuracy measures. The worst performance concerning the accuracy of the smoothed estimator is shown for $\beta=0.9$, in which the high rates of precision errors affect spectrum estimations. The increase in accuracy measures suggests that such value must be avoided in the calculation of robust estimations of spectral density in ARMA 
Table 1

Accuracy measures for the smoothed periodogram: $\operatorname{ARMA}(1,1)$ process with $\phi=0.5$ and $\theta=0.3$

\begin{tabular}{|c|c|c|c|c|c|}
\hline \multicolumn{6}{|c|}{$\beta=0.7$} \\
\hline$\gamma$ & measures & Parzen & $T H$ & Bartlett & Truncated \\
\hline \multirow[t]{3}{*}{0.00} & $R M S E$ & 0.0212 & 0.0281 & 0.0292 & 0.0440 \\
\hline & $M A E$ & 0.0117 & 0.0150 & 0.0163 & 0.0228 \\
\hline & $M A P E$ & 6.5394 & 7.2596 & 9.9014 & 9.8748 \\
\hline \multirow[t]{3}{*}{0.05} & $R M S E$ & 0.0936 & 0.0995 & 0.0911 & 0.1125 \\
\hline & $M A E$ & 0.0607 & 0.0623 & 0.0584 & 0.0679 \\
\hline & $M A P E$ & 23.9648 & 24.4735 & 21.2728 & 27.1426 \\
\hline \multirow[t]{3}{*}{0.10} & $R M S E$ & 0.2234 & 0.2267 & 0.2173 & 0.2368 \\
\hline & $M A E$ & 0.1476 & 0.1487 & 0.1437 & 0.1519 \\
\hline & $M A P E$ & 56.8197 & 57.3552 & 53.1758 & 59.4265 \\
\hline \multirow[t]{3}{*}{0.25} & $R M S E$ & 0.7888 & 0.7962 & 0.7771 & 0.8200 \\
\hline & $M A E$ & 0.5146 & 0.5167 & 0.5070 & 0.5268 \\
\hline & $M A P E$ & 172.5879 & 173.2934 & 165.1584 & 181.3773 \\
\hline \multicolumn{6}{|c|}{$\beta=0.8$} \\
\hline$\gamma$ & measures & Parzen & $T H$ & Bartlett & Truncated \\
\hline \multirow[t]{3}{*}{0.00} & $R M S E$ & 0.0304 & 0.0354 & 0.0328 & 0.0487 \\
\hline & $M A E$ & 0.0149 & 0.0174 & 0.0156 & 0.0258 \\
\hline & $M A P E$ & 6.0567 & 7.3314 & 6.9163 & 11.1520 \\
\hline \multirow[t]{3}{*}{0.05} & $R M S E$ & 0.1014 & 0.1063 & 0.0993 & 0.1200 \\
\hline & $M A E$ & 0.0631 & 0.0652 & 0.0623 & 0.0702 \\
\hline & $M A P E$ & 24.6493 & 25.3046 & 23.3842 & 28.2097 \\
\hline \multirow[t]{3}{*}{0.10} & $R M S E$ & 0.2280 & 0.2325 & 0.2256 & 0.2478 \\
\hline & $M A E$ & 0.1491 & 0.1505 & 0.1472 & 0.1557 \\
\hline & $M A P E$ & 57.5332 & 58.1971 & 55.5902 & 61.2707 \\
\hline \multirow[t]{3}{*}{0.25} & $R M S E$ & 0.7993 & 0.8103 & 0.7963 & 0.8439 \\
\hline & $M A E$ & 0.5176 & 0.5214 & 0.5146 & 0.5350 \\
\hline & $M A P E$ & 173.3536 & 175.2932 & 170.0304 & 185.2228 \\
\hline \multicolumn{6}{|c|}{$\beta=0.9$} \\
\hline$\gamma$ & measures & Parzen & $T H$ & Bartlett & Truncated \\
\hline \multirow[t]{3}{*}{0.00} & $R M S E$ & 0.0357 & 0.0393 & 0.0353 & 0.0644 \\
\hline & $M A E$ & 0.0181 & 0.0216 & 0.0195 & 0.0370 \\
\hline & $M A P E$ & 8.0385 & 9.6417 & 9.2577 & 15.2175 \\
\hline \multirow[t]{3}{*}{0.05} & $R M S E$ & 0.4539 & 0.4558 & 0.4542 & 0.4630 \\
\hline & $M A E$ & 0.4439 & 0.4448 & 0.4446 & 0.4461 \\
\hline & $M A P E$ & 416.6903 & 416.7427 & 418.0991 & 416.9504 \\
\hline \multirow[t]{3}{*}{0.10} & $R M S E$ & 0.4867 & 0.4898 & 0.4869 & 0.4981 \\
\hline & $M A E$ & 0.4436 & 0.4447 & 0.4443 & 0.4469 \\
\hline & $M A P E$ & 335.5855 & 335.5932 & 337.3849 & 335.9566 \\
\hline \multirow[t]{3}{*}{0.25} & $R M S E$ & 0.8150 & 0.8283 & 0.8156 & 0.8658 \\
\hline & $M A E$ & 0.5231 & 0.5282 & 0.5227 & 0.5444 \\
\hline & $M A P E$ & 176.2388 & 179.0922 & 174.7544 & 197.0127 \\
\hline
\end{tabular}


Figure 1

Mean of smoothed periodograms with $\beta=0.7: 10,000$ observations of size 300 of an $\operatorname{ARMA}(1,1)$ process with $\phi=0.5$ and $\theta=0.3$

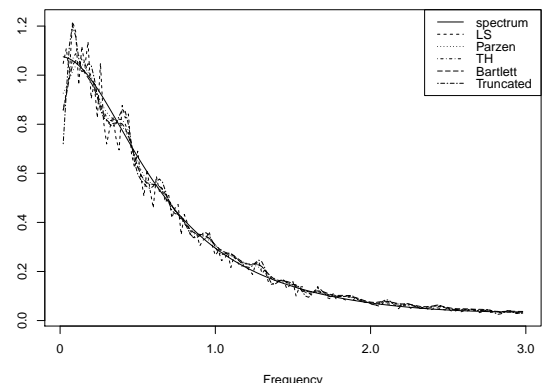

$\gamma=0.0$

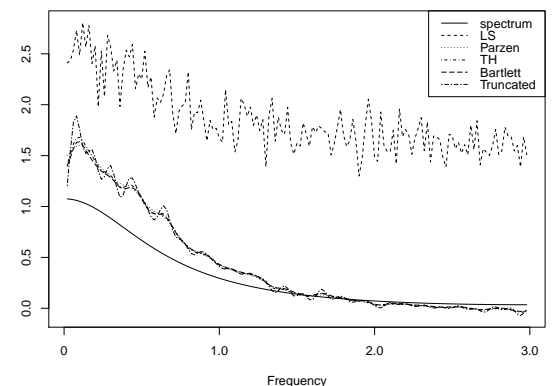

$\gamma=0.10$

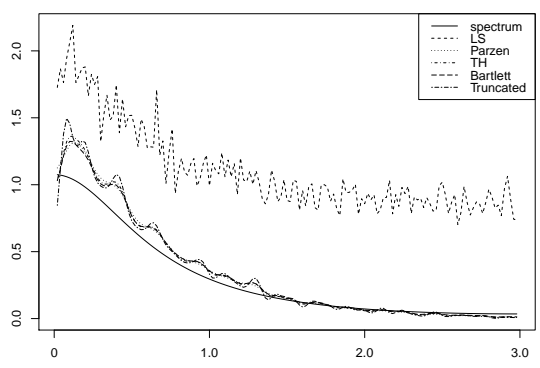

$\gamma=0.05$

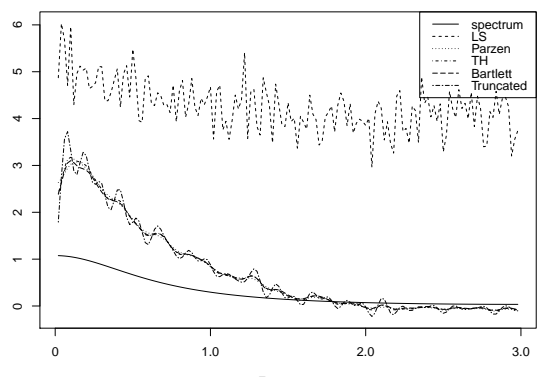

$\gamma=0.25$ 
processes. For contamination rates greater than or equal to $10 \%$, the smoothed periodogram demonstrates a significant increase in accuracy measures, i.e., the properties of the estimator are seriously affected by high rates of contamination.

In uncontaminated series, the ARE of the estimator for Parzen, $T H$ and Bartlett windows is approximately $60 \%$, with a higher efficiency for frequencies closer to zero (see Figures 2 and 3). Among the applied windows, the truncated one renders the smoothed estimator less efficient when different values of $\gamma$ are considered. Results indicate that, for $\gamma=0.25$, the ARE of the estimator is around $2 \%$, making it improper for the spectrum estimation of stationary processes with high contamination rates.

Figure 2

Asymptotic relative efficiency of the smoothed periodogram with $\beta=0.7: \operatorname{ARMA}(1,1)$ process with $\phi=0.5$ and $\theta=0.3$
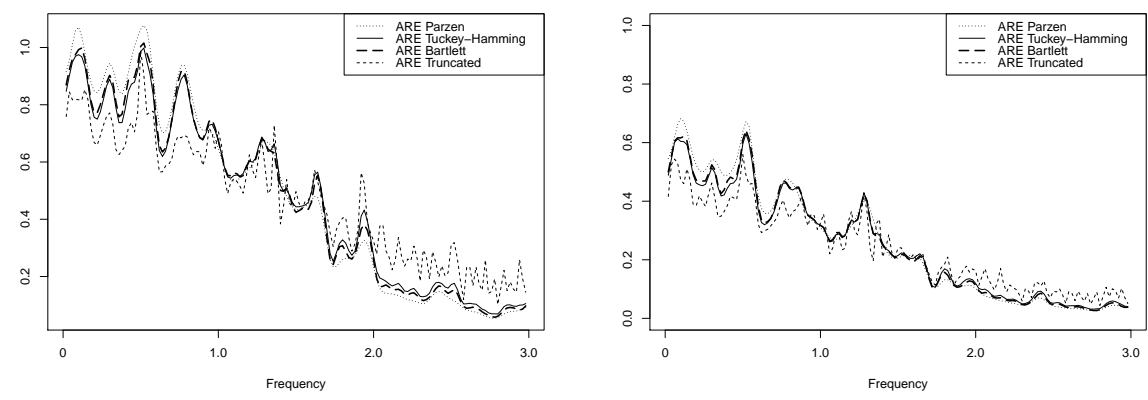

$\gamma=0.0$

$\gamma=0.05$
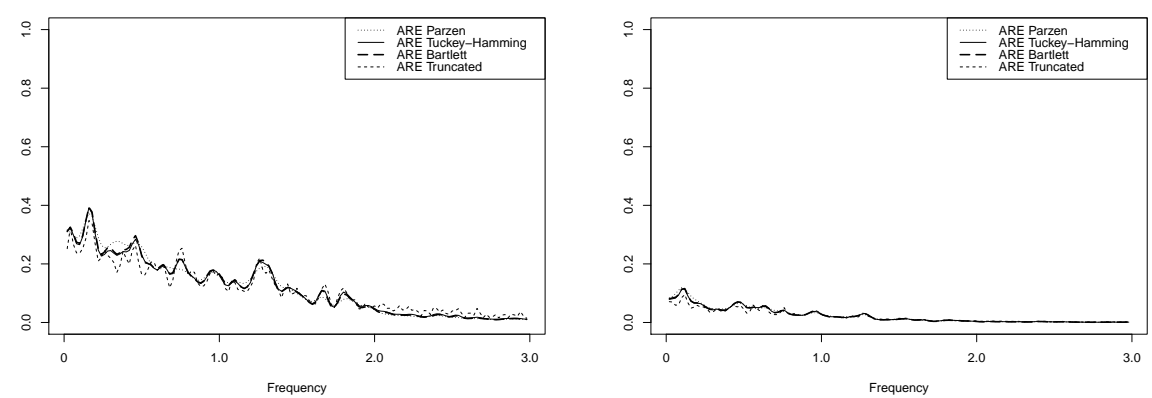

$\gamma=0.10$

$\gamma=0.25$

In the case of ARFIMA $(0,0.3,0)$ process, the smoothed estimator with $T H$ and truncated windows have a higher accuracy when parameter $\beta$ value is 0.7 , with a slight increase in accuracy measures when $\beta=0.8$. The differences between the 
Figure 3

Asymptotic relative efficiency of the smoothed periodogram with $\beta=0.8: \operatorname{ARMA}(1,1)$ process with $\phi=0.5$ and $\theta=0.3$

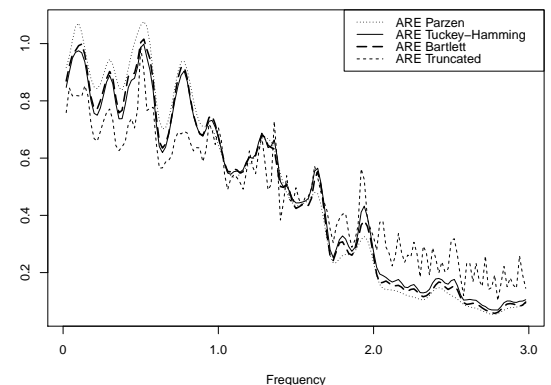

$\gamma=0.0$

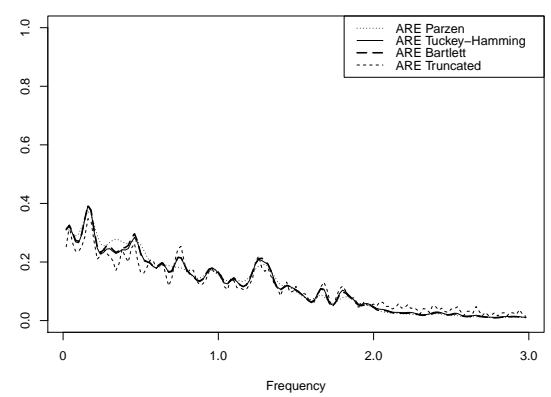

$\gamma=0.10$

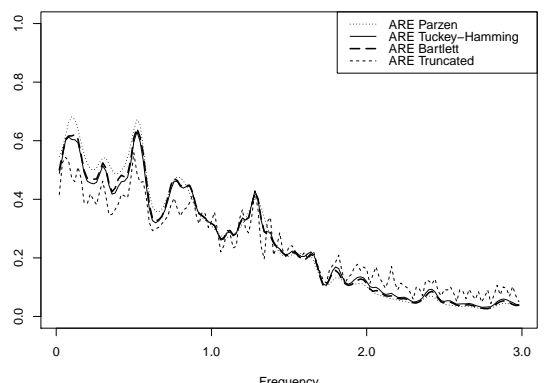

$\gamma=0.05$

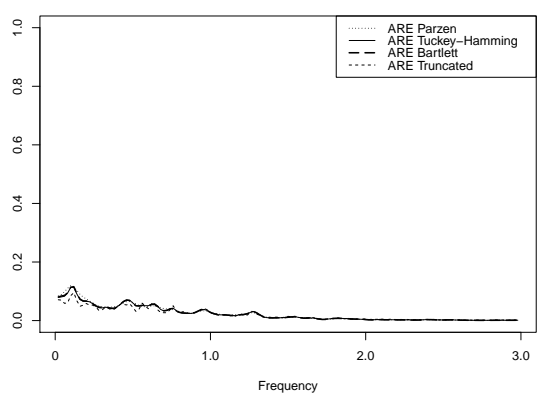

$\gamma=0.25$ 
performance of the estimator, when a smoothing window is used, are not significant for bandwidth values calculated with $\beta=0.7$ or $\beta=0.8$, i.e., there are no significant differences in accuracy measures calculated for different parameter $\beta$ values, but the truncated window is not the best option for the smoothed periodogram (see Table 2). Results indicate that the statistical properties of the robust smoothed periodogram improve significantly because of the spectral window used.

The resistance of estimators to the presence of atypical data in the time series does not depend on the type of window used in the periodogram, but rather on the frequencies used for its calculation; therefore, the sample breakdown point is not influenced by the type of window applied to the robust estimator. Empirically, the PQ value is lower than $10 \%$, since the accuracy of estimators is seriously affected as contamination level rises (see Figures 4, 5 and 6).

Figure 4

Mean of periodograms with $\beta=0.7: 10,000$ observations of size 300 of an $\operatorname{ARFIMA}(0,0.3,0)$ process

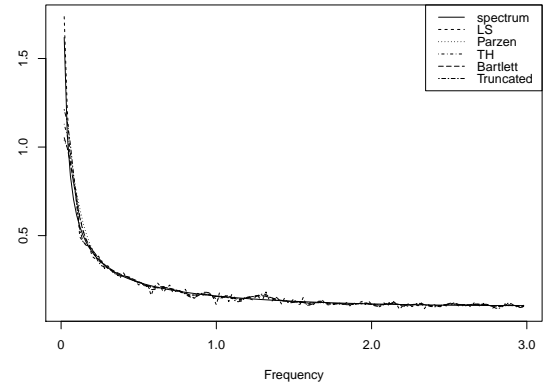

$\gamma=0.0$

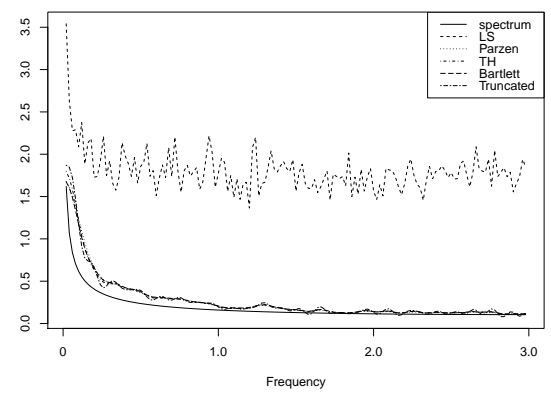

$\gamma=0.10$

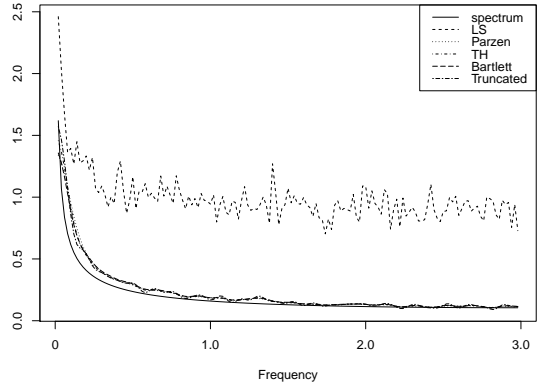

$\gamma=0.05$

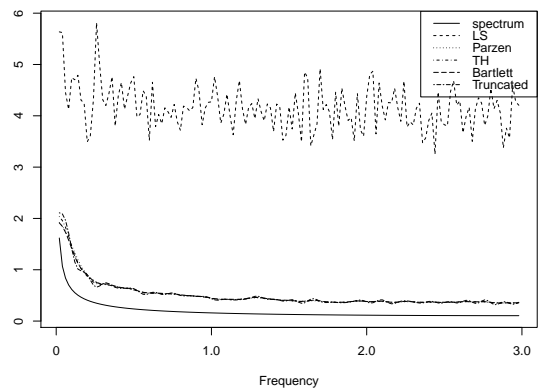

$\gamma=0.25$ 
Table 2

Accuracy measures for the smoothed periodogram: $\operatorname{ARFIMA}(0,0.3,0)$ process

\begin{tabular}{|c|c|c|c|c|c|}
\hline \multicolumn{6}{|c|}{$\beta=0.7$} \\
\hline$\gamma$ & measures & Parzen & $T H$ & Bartlett & Truncated \\
\hline \multirow[t]{3}{*}{0.00} & $R M S E$ & 0.0522 & 0.0446 & 0.0488 & 0.0403 \\
\hline & $M A E$ & 0.0120 & 0.0111 & 0.0109 & 0.0139 \\
\hline & $M A P E$ & 3.4747 & 3.9750 & 3.5849 & 6.1065 \\
\hline \multirow[t]{3}{*}{0.05} & $R M S E$ & 0.0767 & 0.0734 & 0.0693 & 0.0730 \\
\hline & $M A E$ & 0.0405 & 0.0385 & 0.0401 & 0.0377 \\
\hline & $M A P E$ & 16.1522 & 15.7194 & 17.0576 & 16.3730 \\
\hline \multirow[t]{3}{*}{0.10} & $R M S E$ & 0.1430 & 0.1453 & 0.1361 & 0.1524 \\
\hline & $M A E$ & 0.0790 & 0.0785 & 0.0789 & 0.0801 \\
\hline & $M A P E$ & 32.8495 & 32.3349 & 34.0494 & 33.5176 \\
\hline \multirow[t]{3}{*}{0.25} & $R M S E$ & 0.3405 & 0.3412 & 0.3376 & 0.3441 \\
\hline & $M A E$ & 0.3189 & 0.3185 & 0.3189 & 0.3183 \\
\hline & $M A P E$ & 200.4568 & 199.9444 & 201.6625 & 199.5148 \\
\hline \multicolumn{6}{|c|}{$\beta=0.8$} \\
\hline$\gamma$ & measures & Parzen & $T H$ & Bartlett & Truncated \\
\hline \multirow[t]{3}{*}{0.00} & $R M S E$ & 0.0433 & 0.0412 & 0.0440 & 0.0505 \\
\hline & $M A E$ & 0.0112 & 0.0124 & 0.0113 & 0.0218 \\
\hline & $M A P E$ & 4.2429 & 5.0965 & 4.4831 & 9.3537 \\
\hline \multirow[t]{3}{*}{0.05} & $R M S E$ & 0.0730 & 0.0742 & 0.0709 & 0.0912 \\
\hline & $M A E$ & 0.0383 & 0.0381 & 0.0391 & 0.0412 \\
\hline & $M A P E$ & 15.7755 & 15.9503 & 16.5413 & 18.0506 \\
\hline \multirow[t]{3}{*}{0.10} & $R M S E$ & 0.1460 & 0.1498 & 0.1429 & 0.1630 \\
\hline & $M A E$ & 0.0785 & 0.0789 & 0.0790 & 0.0839 \\
\hline & $M A P E$ & 32.2739 & 32.3974 & 33.2495 & 35.8455 \\
\hline \multirow[t]{3}{*}{0.25} & $R M S E$ & 0.5165 & 0.5268 & 0.5123 & 0.5823 \\
\hline & $M A E$ & 0.3134 & 0.3147 & 0.3148 & 0.3235 \\
\hline & $M A P E$ & 136.4854 & 136.8104 & 138.5574 & 140.4886 \\
\hline \multicolumn{6}{|c|}{$\beta=0.9$} \\
\hline$\gamma$ & measures & Parzen & $T H$ & Bartlett & Truncated \\
\hline \multirow[t]{3}{*}{0.00} & $R M S E$ & 0.0402 & 0.0376 & 0.0376 & 0.0354 \\
\hline & $M A E$ & 0.0129 & 0.0148 & 0.0135 & 0.0201 \\
\hline & $M A P E$ & 5.4695 & 6.7888 & 6.0912 & 11.1038 \\
\hline \multirow[t]{3}{*}{0.05} & $R M S E$ & 0.0750 & 0.0788 & 0.0745 & 0.0894 \\
\hline & $M A E$ & 0.0380 & 0.0390 & 0.0384 & 0.0456 \\
\hline & $M A P E$ & 16.0405 & 16.6804 & 16.6406 & 20.6241 \\
\hline \multirow[t]{3}{*}{0.10} & $R M S E$ & 0.1513 & 0.1558 & 0.1500 & 0.1676 \\
\hline & $M A E$ & 0.0795 & 0.0816 & 0.0810 & 0.0892 \\
\hline & $M A P E$ & 32.7155 & 33.8761 & 33.8776 & 39.2540 \\
\hline \multirow[t]{3}{*}{0.25} & $R M S E$ & 0.5340 & 0.5581 & 0.5441 & 0.6149 \\
\hline & $M A E$ & 0.3160 & 0.3198 & 0.3189 & 0.3360 \\
\hline & $M A P E$ & 137.0726 & 137.8391 & 138.3392 & 146.7352 \\
\hline
\end{tabular}


Figure 5

Mean of periodograms with $\beta=0.8: 10,000$ observations of size 300 of an $\operatorname{ARFIMA}(0,0.3,0)$ process

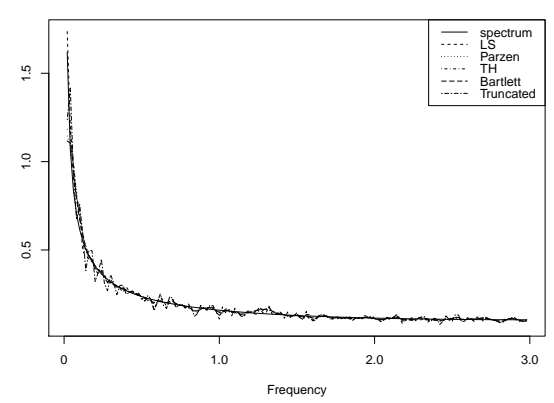

$\gamma=0.0$

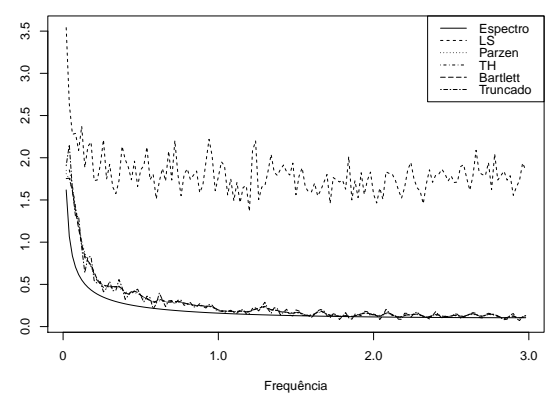

$\gamma=0.10$

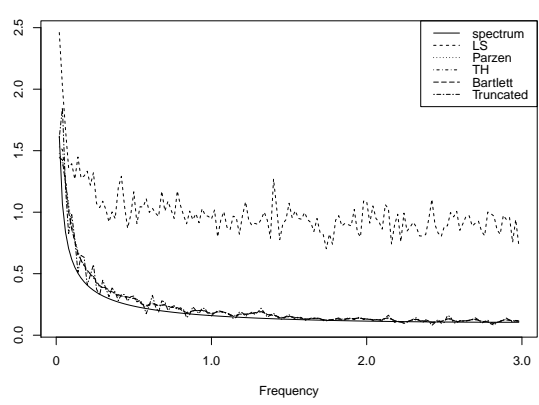

$\gamma=0.05$

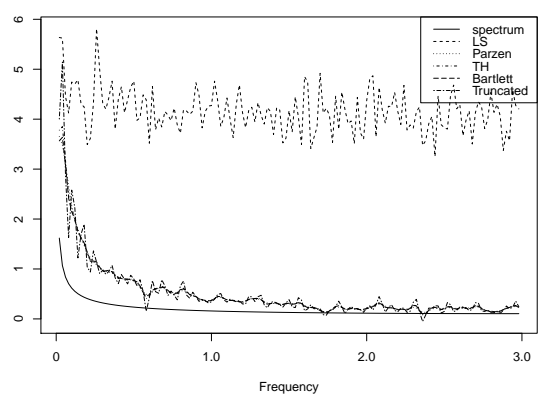

$\gamma=0.25$

The relative efficiency of the estimator is higher when the process follows an ARFIMA representation. ARE values are close to $65 \%$ when the process is free of contamination. The estimator with the truncated window has the lowest ARE values, suggesting that Parzen, TH or Bartlett windows can improve the efficiency of the robust estimator. In the case of uncontaminated series, Figures 7 and 8 reveal a significantly lower efficiency for the estimator when the contamination level increases in the set of observations. Nevertheless, ARE values are higher in ARFIMA processes, making the estimator statistically more efficient in the spectrum estimation of long-memory processes.

As with ARMA, the increase in the smoothed estimator's variance results from the Fourier frequencies, showing an increase in variances for frequencies close to $\pi$ and suggesting higher efficiency for frequencies around zero. Therefore, Tables 3 and 4 display the accuracy measures for the first $n^{\alpha}$ frequencies, with $\alpha=0.5$ and 0.7 and a sample size of 300. The values chosen for $\alpha$ are commonly used to 
Figure 6

Mean of periodograms with $\beta=0.9: 10,000$ observations of size 300 of an $\operatorname{ARFIMA}(0,0.3,0)$ process

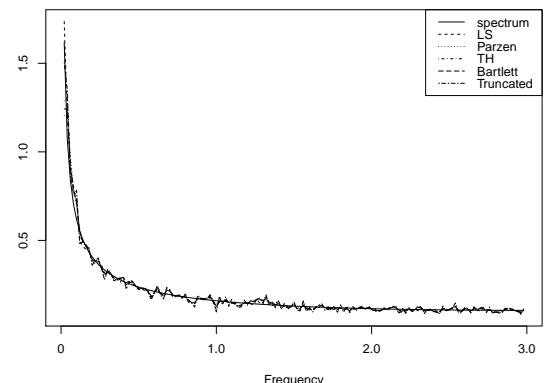

$\gamma=0.0$

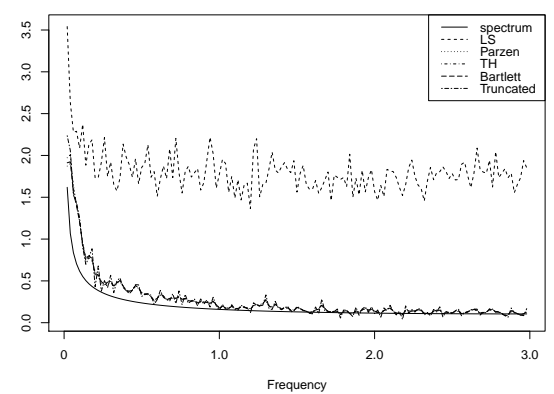

$\gamma=0.10$

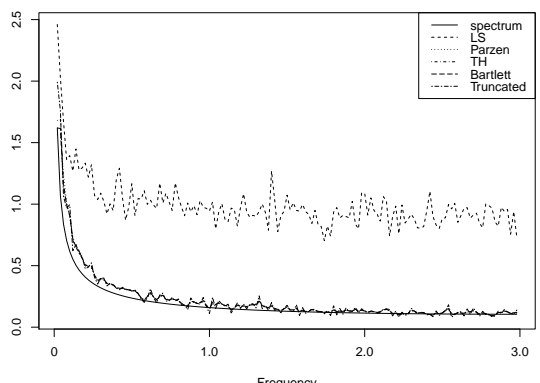

$\gamma=0.05$

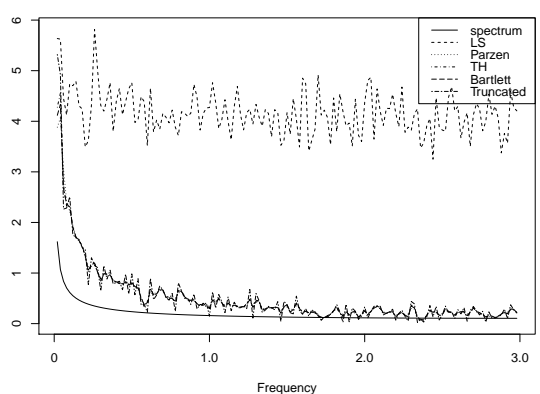

$\gamma=0.25$ 
estimate the fractional differencing parameter in $\operatorname{ARFIMA}(p, d, q)$ models through the LP estimator suggested by Geweke and Porter-Hudak (1983). Table 3 presents the results for the first 17 frequencies. The results indicate an increase in precision errors, suggesting better estimator performance when the Bartlett window is used. The same result is shown in Table 4, where the first 54 frequencies are considered. Parzen window turns out to be the second best option amongst the windows applied to the robust estimator. Parzen and Bartlett windows can be considered the best alternatives to improve the performance of the robust smoothed periodogram.

Figure 7

Asymptotic relative efficiency for the smoothed periodogram with $\beta=0.7$ :

$\operatorname{ARFIMA}(0,0.3,0)$ process

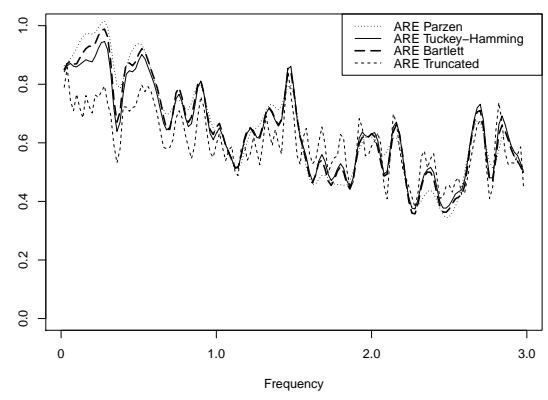

$\gamma=0.0$

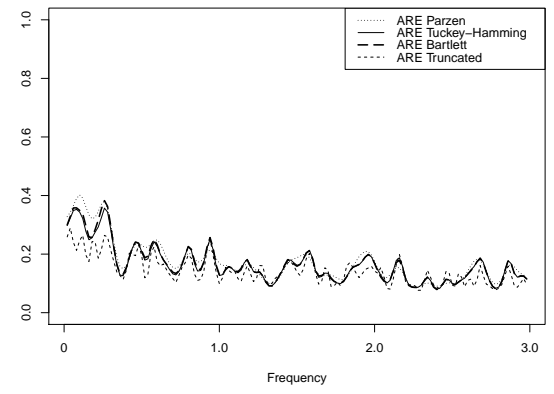

$\gamma=0.10$

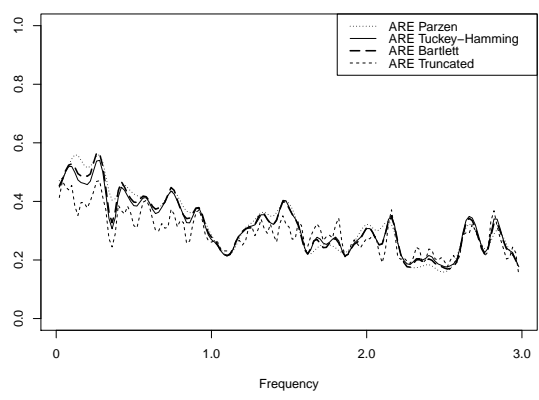

$\gamma=0.05$

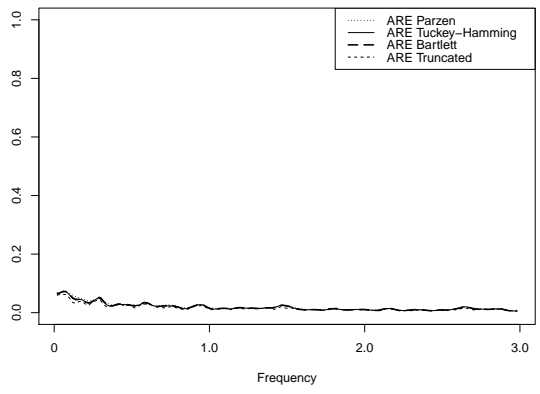

$\gamma=0.25$ 
Table 3

Accuracy measures: $\operatorname{ARFIMA}(0,0.3,0)$ process with $\alpha=0.5$.

\begin{tabular}{|c|c|c|c|c|c|}
\hline \multicolumn{6}{|c|}{$\beta=0.7$} \\
\hline$\gamma$ & measures & Parzen & $T H$ & Bartlett & Truncated \\
\hline \multirow[t]{3}{*}{0.00} & $R M S E$ & 0.1539 & 0.1308 & 0.1435 & 0.1159 \\
\hline & $M A E$ & 0.0767 & 0.0588 & 0.0616 & 0.0588 \\
\hline & $M A P E$ & 9.1784 & 6.5464 & 6.6658 & 6.8942 \\
\hline \multirow[t]{3}{*}{0.05} & $R M S E$ & 0.2151 & 0.2049 & 0.1891 & 0.2015 \\
\hline & $M A E$ & 0.1894 & 0.1740 & 0.1697 & 0.1583 \\
\hline & $M A P E$ & 34.6163 & 31.3014 & 31.3419 & 28.2852 \\
\hline \multirow[t]{3}{*}{0.10} & $R M S E$ & 0.3897 & 0.3967 & 0.3632 & 0.4166 \\
\hline & $M A E$ & 0.3356 & 0.3345 & 0.3147 & 0.3357 \\
\hline & $M A P E$ & 64.3687 & 61.2932 & 60.6496 & 59.3647 \\
\hline \multirow[t]{3}{*}{0.25} & $R M S E$ & 0.6087 & 0.6127 & 0.5835 & 0.6264 \\
\hline & $M A E$ & 0.5756 & 0.5744 & 0.5547 & 0.5757 \\
\hline & $M A P E$ & 117.2539 & 114.1767 & 113.5346 & 112.2504 \\
\hline \multicolumn{6}{|c|}{$\beta=0.8$} \\
\hline$\gamma$ & measures & Parzen & $T H$ & Bartlett & Truncated \\
\hline \multirow[t]{3}{*}{0.00} & $R M S E$ & 0.1267 & 0.1196 & 0.1286 & 0.1427 \\
\hline & $M A E$ & 0.0565 & 0.0577 & 0.0530 & 0.0969 \\
\hline & $M A P E$ & 6.1389 & 6.4098 & 5.4293 & 15.1302 \\
\hline \multirow[t]{3}{*}{0.05} & $R M S E$ & 0.2032 & 0.2062 & 0.1946 & 0.2532 \\
\hline & $M A E$ & 0.1711 & 0.1661 & 0.1676 & 0.1658 \\
\hline & $M A P E$ & 30.7182 & 29.4179 & 30.2294 & 28.4710 \\
\hline \multirow[t]{3}{*}{0.10} & $R M S E$ & 0.3987 & 0.4094 & 0.3857 & 0.4422 \\
\hline & $M A E$ & 0.3342 & 0.3354 & 0.3244 & 0.3413 \\
\hline & $M A P E$ & 60.7756 & 59.5652 & 59.8609 & 58.3672 \\
\hline \multirow[t]{3}{*}{0.25} & $R M S E$ & 1.3795 & 1.4099 & 1.3588 & 1.5719 \\
\hline & $M A E$ & 1.2476 & 1.2540 & 1.2306 & 1.2893 \\
\hline & $M A P E$ & 229.0855 & 227.0410 & 227.0897 & 226.9018 \\
\hline \multicolumn{6}{|c|}{$\beta=0.9$} \\
\hline$\gamma$ & measures & Parzen & $T H$ & Bartlett & Truncated \\
\hline \multirow[t]{3}{*}{0.00} & $R M S E$ & 0.1160 & 0.1061 & 0.1071 & 0.0899 \\
\hline & $M A E$ & 0.0567 & 0.0584 & 0.0543 & 0.0556 \\
\hline & $M A P E$ & 6.2243 & 7.1638 & 6.4036 & 8.7392 \\
\hline \multirow[t]{3}{*}{0.05} & $R M S E$ & 0.2085 & 0.2184 & 0.2050 & 0.2441 \\
\hline & $M A E$ & 0.1650 & 0.1652 & 0.1595 & 0.1802 \\
\hline & $M A P E$ & 29.2578 & 28.8540 & 29.0396 & 29.2921 \\
\hline \multirow[t]{3}{*}{0.10} & $R M S E$ & 0.4134 & 0.4251 & 0.4065 & 0.4516 \\
\hline & $M A E$ & 0.3373 & 0.3430 & 0.3362 & 0.3559 \\
\hline & $M A P E$ & 59.5145 & 59.2707 & 59.5946 & 59.5758 \\
\hline \multirow[t]{3}{*}{0.25} & $R M S E$ & 1.4320 & 1.5040 & 1.4587 & 1.6567 \\
\hline & $M A E$ & 1.2636 & 1.2903 & 1.2737 & 1.3431 \\
\hline & $M A P E$ & 227.4940 & 228.1230 & 228.2778 & 229.6183 \\
\hline
\end{tabular}


Table 4

Accuracy measures: ARFIMA $(0,0.3,0)$ process with $\alpha=0.7$.

\begin{tabular}{|c|c|c|c|c|c|}
\hline \multicolumn{6}{|c|}{$\beta=0.7$} \\
\hline$\gamma$ & measures & Parzen & $T H$ & Bartlett & Truncated \\
\hline \multirow[t]{3}{*}{0.00} & $R M S E$ & 0.0864 & 0.0736 & 0.0806 & 0.0657 \\
\hline & $M A E$ & 0.0264 & 0.0218 & 0.0225 & 0.0246 \\
\hline & $M A P E$ & 4.0724 & 3.7724 & 3.6521 & 5.4099 \\
\hline \multirow[t]{3}{*}{0.05} & $R M S E$ & 0.1254 & 0.1197 & 0.1125 & 0.1183 \\
\hline & $M A E$ & 0.0863 & 0.0809 & 0.0827 & 0.0759 \\
\hline & $M A P E$ & 23.8718 & 22.6194 & 24.1507 & 21.7428 \\
\hline \multirow[t]{3}{*}{0.10} & $R M S E$ & 0.2340 & 0.2375 & 0.2216 & 0.2481 \\
\hline & $M A E$ & 0.1702 & 0.1692 & 0.1666 & 0.1688 \\
\hline & $M A P E$ & 51.4336 & 50.1171 & 51.7112 & 49.1358 \\
\hline \multirow[t]{3}{*}{0.25} & $R M S E$ & 0.4405 & 0.4418 & 0.4321 & 0.4474 \\
\hline & $M A E$ & 0.4102 & 0.4092 & 0.4066 & 0.4087 \\
\hline & $M A P E$ & 152.8701 & 151.5539 & 153.1523 & 150.5752 \\
\hline \multicolumn{6}{|c|}{$\beta=0.8$} \\
\hline$\gamma$ & measures & Parzen & TH & Bartlett & Truncated \\
\hline \multirow[t]{3}{*}{0.00} & $R M S E$ & 0.0713 & 0.0676 & 0.0725 & 0.0822 \\
\hline & $M A E$ & 0.0213 & 0.0227 & 0.0208 & 0.0426 \\
\hline & $M A P E$ & 3.8054 & 4.4577 & 3.8628 & 10.9773 \\
\hline \multirow[t]{3}{*}{0.05} & $R M S E$ & 0.1189 & 0.1207 & 0.1150 & 0.1484 \\
\hline & $M A E$ & 0.0800 & 0.0784 & 0.0806 & 0.0823 \\
\hline & $M A P E$ & 22.4634 & 22.0488 & 23.1535 & 23.7475 \\
\hline \multirow[t]{3}{*}{0.10} & $R M S E$ & 0.2387 & 0.2446 & 0.2329 & 0.2647 \\
\hline & $M A E$ & 0.1691 & 0.1695 & 0.1681 & 0.1726 \\
\hline & $M A P E$ & 49.9461 & 49.5326 & 50.6802 & 49.6572 \\
\hline \multirow[t]{3}{*}{0.25} & $R M S E$ & 0.8387 & 0.8554 & 0.8304 & 0.9453 \\
\hline & $M A E$ & 0.6473 & 0.6494 & 0.6462 & 0.6638 \\
\hline & $M A P E$ & 196.8789 & 196.2961 & 198.3645 & 197.7665 \\
\hline \multicolumn{6}{|c|}{$\beta=0.9$} \\
\hline$\gamma$ & measures & Parzen & $T H$ & Bartlett & Truncated \\
\hline \multirow[t]{3}{*}{0.00} & $R M S E$ & 0.0656 & 0.0608 & 0.0611 & 0.0543 \\
\hline & $M A E$ & 0.0233 & 0.0259 & 0.0238 & 0.0307 \\
\hline & $M A P E$ & 4.8024 & 6.1278 & 5.4475 & 9.6817 \\
\hline \multirow[t]{3}{*}{0.05} & $R M S E$ & 0.1220 & 0.1279 & 0.1208 & 0.1433 \\
\hline & $M A E$ & 0.0780 & 0.0790 & 0.0776 & 0.0867 \\
\hline & $M A P E$ & 21.9987 & 22.4037 & 22.5783 & 24.2550 \\
\hline \multirow[t]{3}{*}{0.10} & $R M S E$ & 0.2469 & 0.2538 & 0.2441 & 0.2701 \\
\hline & $M A E$ & 0.1702 & 0.1722 & 0.1712 & 0.1769 \\
\hline & $M A P E$ & 49.5408 & 49.5662 & 50.2626 & 50.0216 \\
\hline \multirow[t]{3}{*}{0.25} & $R M S E$ & 0.8671 & 0.9061 & 0.8828 & 0.9942 \\
\hline & $M A E$ & 0.6523 & 0.6610 & 0.6581 & 0.6796 \\
\hline & $M A P E$ & 196.4488 & 196.8441 & 198.0045 & 198.3488 \\
\hline
\end{tabular}


Figure 8

Asymptotic relative efficiency for the smoothed periodogram with $\beta=0.8$ : $\operatorname{ARFIMA}(0,0.3,0)$ process

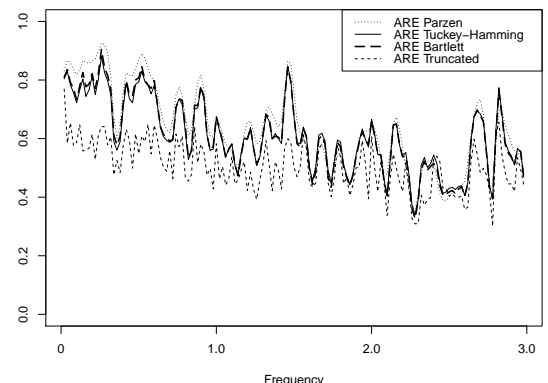

$\gamma=0.0$

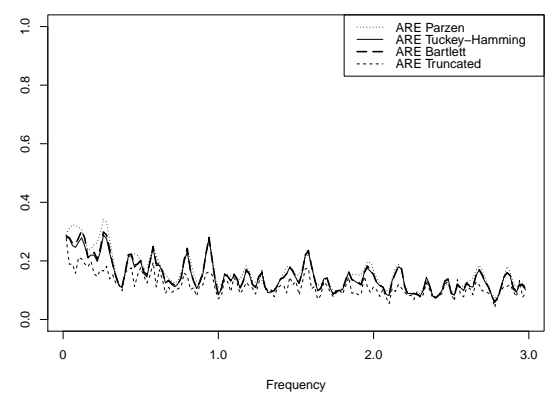

$\gamma=0.10$

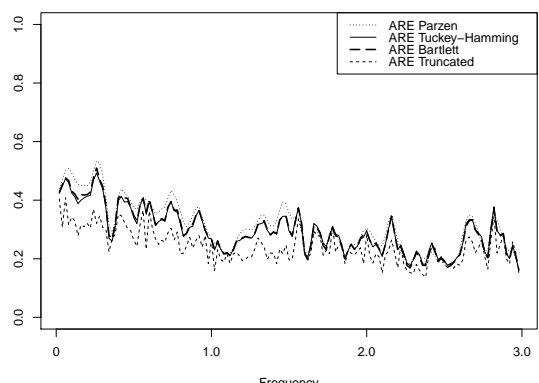

$\gamma=0.05$

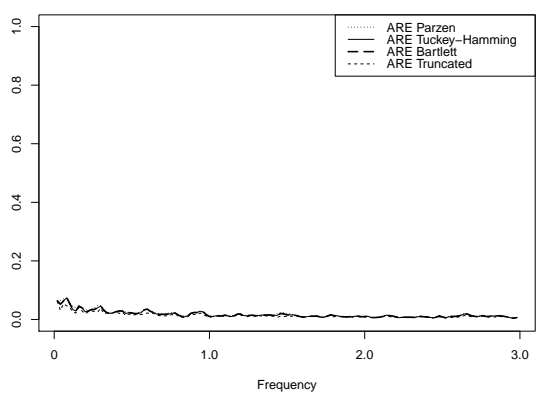

$\gamma=0.25$ 


\subsection{Application: IGP-DI series data}

IGP-DI stands for general price index with domestic availability, and is calculated by Getulio Vargas Foundation. The sampling period went from August 1994 to April 2011 (201 observations). Figure 9 demonstrates the dynamics of the time series with its respective autocorrelation functions (ACF), partial autocorrelation function (PACF) and periodogram. The observations referring to February 1999 (4.44) and November 2002 (5.84) can increase data variance, affecting the parameter estimation of the fitted model. The ACF has statistically significant correlations for distant lags; moreover, the periodogram yields the highest values for near-zero frequencies, suggesting the presence of long memory in observations. To assess the behavior of robust estimators in real data, an outlier is induced in the dataset. The observation referring to November 2002 is replaced with the value of 24.13 , which is the result of the sum of the original value (5.84) and 20 times the standard deviation of data (0.9146). Figure 10 shows the contaminated IGP-DI series and its respective ACF, PACF and periodogram function. The ACF of the contaminated series shows a decrease in autocorrelation estimation values and a significant increase in the periodogram values, which seriously affects the estimates obtained from classic methods.

Assuming that the IGP-DI series was generated from an $\operatorname{ARFIMA}(0, d, 0)$ process, and in order to analyze the behavior of robust periodograms defined in Eqs. 6 and 10, estimations for the fractional differencing parameter are made for the series with and without contamination. Table 5 displays the estimates obtained from semiparametric estimators, with the windows defined in Section 2. Robust estimators are denoted as $d_{P r}, d_{T H r}, d_{B r}$ and $d_{L P R}$, for Parzen, Tuckey-Hamming, Bartlett, and truncated windows, respectively. For bandwidth calculation, values of $\beta=0.9$ were assumed for Parzen window and the value of $\beta=0.7$ for $\mathrm{TH}$ and Bartlett and truncated windows. These values of $\beta$ are selected from the results obtained in the previous section, as they allow maintaining the balance between accuracy and relative efficiency of the smoothed estimator. For the estimators defined in Eqs. 6 and 10, the values of $\alpha$ are defined between 0.5 and 0.8. The values in brackets correspond to the standard deviations of the estimates.

The results shown in Table 5 reveal that the LP estimator is significantly affected in the presence of contaminated data, tending to underestimate the true value of parameter $d$ for any value of $\alpha$. Robust estimators do not vary a lot when different bandwidth values are taken into account, suggesting that the data are generated from a stationary long-memory process with parameter $d=0.2$. The robust estimator estimates when applied to Parzen and Bartlett windows are noteworthy as far as memory parameter estimations are concerned, as they often have the lowest standard deviations. This finding is consistent with the results of Monte Carlo simulations, conducted in the previous section. 
Figure 9

IGP-DI series and autocorrelation functions: period from Aug/94 to Apr/11
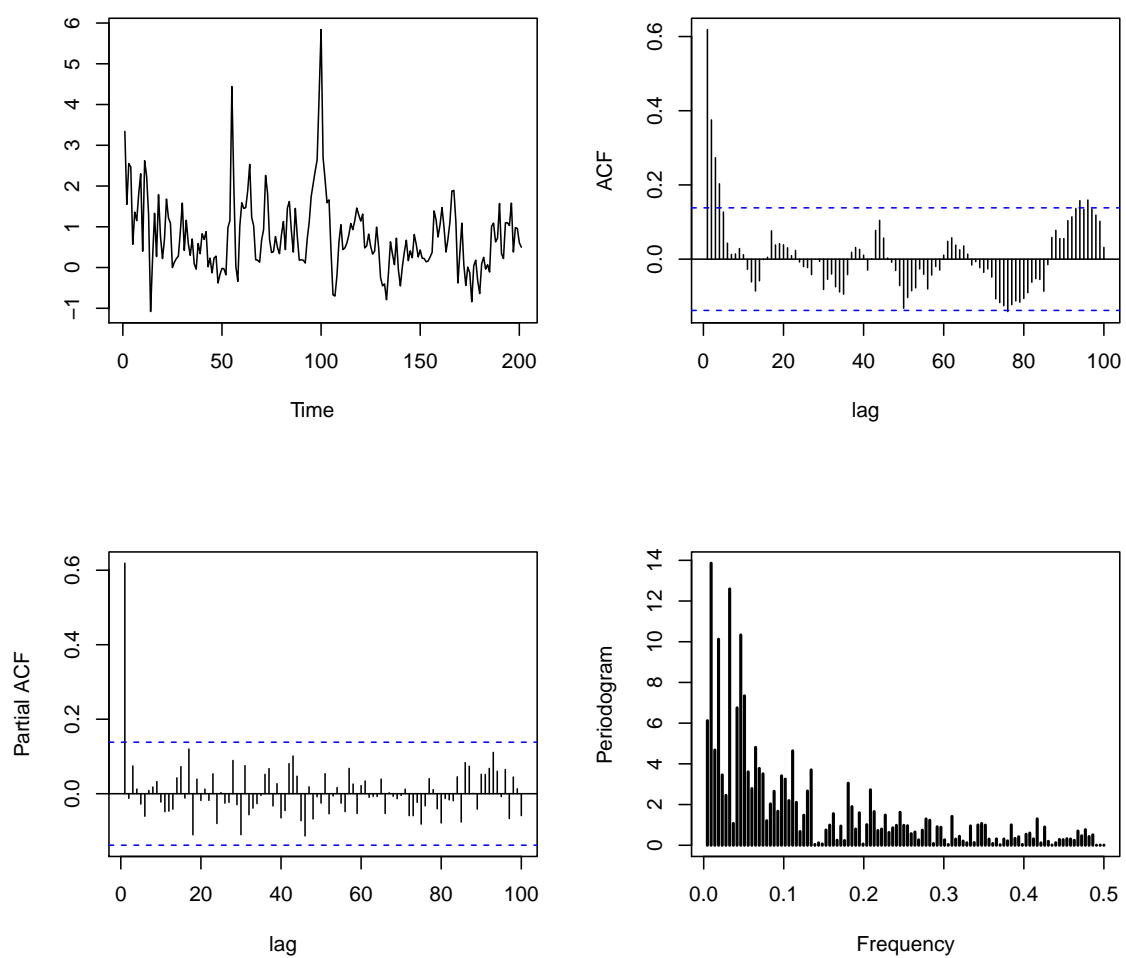

Table 5

Estimates $d$ : IGP-DI data, period from Aug/94 to Apr/11

\begin{tabular}{|c|c|c|c|c|c|c|c|c|}
\hline & \multicolumn{4}{|c|}{ Uncontaminated } & \multicolumn{4}{|c|}{ Contaminated } \\
\hline Estimator & $\alpha=0.5$ & $\alpha=0.6$ & $\alpha=0.7$ & $\alpha=0.8$ & $\alpha=0.5$ & $\alpha=0.6$ & $\alpha=0.7$ & $\alpha=0.8$ \\
\hline$d_{L P}$ & $\begin{array}{c}0.0757 \\
(0.1168)\end{array}$ & $\begin{array}{c}0.1205 \\
(0.0349)\end{array}$ & 0.3431 & $\begin{array}{c}0.3759 \\
(0.0079)\end{array}$ & $\begin{array}{l}-0.0070 \\
(0.0184)\end{array}$ & 0.1710 & 0.2465 & 0.2050 \\
\hline$d_{P}$ & $\begin{array}{c}(0.1168) \\
0.1802\end{array}$ & $\begin{array}{r}(0.034 \\
0.233\end{array}$ & $\begin{array}{r}(0.019 \\
0.226\end{array}$ & $\begin{array}{l}(0.007 \\
0.239\end{array}$ & $\begin{array}{c}(0.0184) \\
0.1804\end{array}$ & $\begin{array}{c}(0.0150) \\
0.2335\end{array}$ & $(0.0070)$ & $\begin{array}{l}(0.0028) \\
0.2398\end{array}$ \\
\hline$a_{P r}$ & $\begin{array}{l}0.1802 \\
(0.0074)\end{array}$ & $\begin{array}{l}0.2335 \\
(0.0056)\end{array}$ & $\begin{array}{l}0.269 \\
(0.0023)\end{array}$ & $\begin{array}{l}0.2397 \\
(0.0011)\end{array}$ & $\begin{array}{l}0.1804 \\
(0.0073)\end{array}$ & $\begin{array}{l}0.2335 \\
(0.0055)\end{array}$ & $\begin{array}{l}0.2272 \\
(0.0022)\end{array}$ & $(0.0011)$ \\
\hline$d_{T H r}$ & $\begin{array}{c}0.1749 \\
(0.0117)\end{array}$ & $\begin{array}{c}0.2509 \\
(0.0085)\end{array}$ & $\begin{array}{c}0.2319 \\
(0.0037)\end{array}$ & $\begin{array}{c}0.2414 \\
(0.0022)\end{array}$ & $\begin{array}{c}0.1752 \\
(0.0117)\end{array}$ & $\begin{array}{c}0.2517 \\
(0.0086)\end{array}$ & $\begin{array}{c}0.2327 \\
(0.0038)\end{array}$ & $\begin{array}{c}0.2410 \\
(0.0021)\end{array}$ \\
\hline$d_{B r}$ & $\begin{array}{c}0.1606 \\
(0.0094)\end{array}$ & $\begin{array}{c}0.2259 \\
(0.0059)\end{array}$ & $\begin{array}{c}0.2228 \\
(0.0026)\end{array}$ & $\begin{array}{c}0.2364 \\
(0.0014)\end{array}$ & $\begin{array}{c}0.1609 \\
(0.0094)\end{array}$ & $\begin{array}{c}0.2263 \\
(0.0060)\end{array}$ & $\begin{array}{c}0.2234 \\
(0.0027)\end{array}$ & $\begin{array}{c}0.2363 \\
(0.0014)\end{array}$ \\
\hline$d_{L P R}$ & $\begin{array}{c}0.1662 \\
(0.0073)\end{array}$ & $\begin{array}{c}0.2628 \\
(0.0100)\end{array}$ & $\begin{array}{c}0.2454 \\
(0.0045)\end{array}$ & $\begin{array}{c}0.2285 \\
(0.0019)\end{array}$ & $\begin{array}{c}0.1662 \\
(0.0074)\end{array}$ & $\begin{array}{c}0.2628 \\
(0.0099)\end{array}$ & $\begin{array}{c}0.2454 \\
(0.0045)\end{array}$ & $\begin{array}{c}0.2285 \\
(0.0019) \\
\end{array}$ \\
\hline
\end{tabular}


Figure 10

Contaminated IGP-DI series and autocorrelation functions: period from Aug/94 to Apr/11
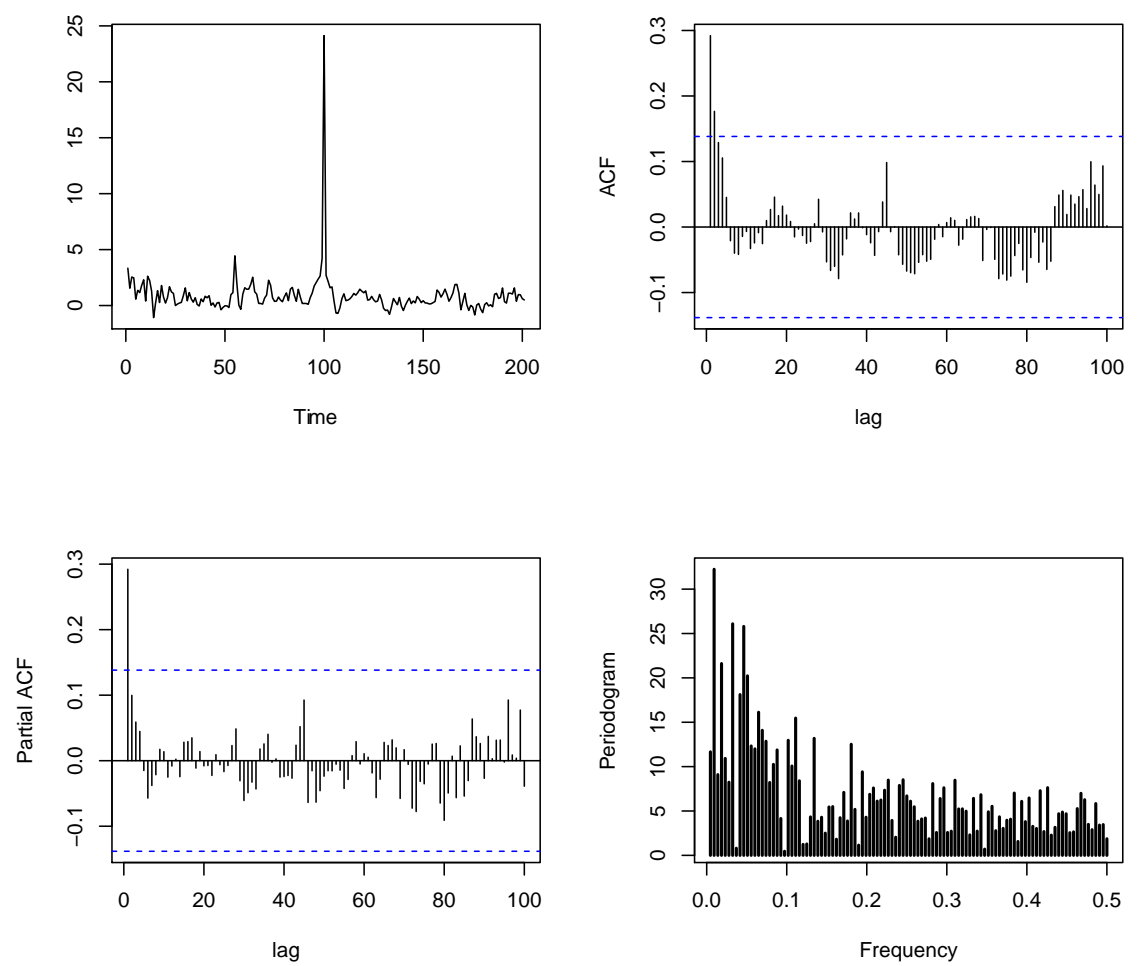


\section{Final Remarks}

This paper analyzes the behavior of the robust pseudoperiodogram proposed by Fajardo et al. (2009) for Parzen, Tuckey-Hamming and Bartlett windows. Empirical studies allow concluding that robust estimators are resistant in time series contaminated by additive outliers. By running Monte Carlo simulations, it is shown that alternative windows improve the statistical properties of the robust pseudoperiodogram. Values of $\beta=0.7$ are suggested for $\mathrm{TH}$ and Bartlett windows and a value of $\beta=0.9$ is suggested for Parzen window as, according to empirical studies, these values allow improving the asymptotic efficiency and the accuracy of estimators in the spectrum estimation of stationary processes.

In addition, the robust periodogram defined in Eq. 3 is used to suggest an estimator for the fractional differencing parameter in stationary ARFIMA processes. The robust estimator for $d$ is a variant of the estimator proposed by Geweke and Porter-Hudak (1983) and defined in Eq. 10. The results indicate that Parzen window with $\beta=0.9$ and Bartlett window with $\beta=0.7$ tend to improve the performance of the estimator $\widehat{f}_{\text {rob }}\left(\lambda_{j}\right)$ defined in Eq. 3, as it tends to have the lowest accuracy measures and higher relative efficiency in series contaminated by atypical additive data.

The statistical properties of the estimators, as well as the definition of an optimal bandwidth in contaminated series, are still an open problem. Recently, the works developed by Lévy-Leduc et al. (2011b) and Lévy-Leduc et al. (2011a) show that the robust estimator of the autocovariance function, suggested by Ma and Genton (2000), satisfies the central limit theorem in the context of stationary processes, but there are no theoretical results that allow establishing the asymptotic properties of the robust estimator of the spectrum.

\section{References}

Anderson, T. W. (1971). The Statistical Analysis of Time Series. John Wiley \& Sons.

Beltrão, K. \& Bloomfield, P. (1987). Determining the bandwidth of a kernel spectrum estimate. Journal of Time Series Analysis, 8:21-38.

Brockwell, P. J. \& Davis, R. A. (2006). Time Series: Theory and Methods. Springer Series in Statistics, second edition.

Chen, G., Abraham, B., \& Peiris, S. (1994). Lag window estimation of the degree of differencing in fractionally integrated time series models. Journal of Time Series Analysis, 15:473-487.

Deo, R. \& Hurvich, C. (2001). On the log periodogram regression estimator of the memory parameter in long memory stochastic volatility models. Econometric Theory, 17:686-710. 
Fajardo, F., Reisen, V. A., \& Cribari-Neto, F. (2009). Robust estimation in longmemory processes under additive outliers. Journal of Statistical Planning and Inference, 139:2511-2525.

Fox, A. J. (1972). Outliers in time series. Journal of the Royal Statistical Society, 34(B):350-363.

Geweke, J. \& Porter-Hudak, S. (1983). The estimation and application of long memory time series model. Journal of Time Series Analysis, 4:221-238.

Haldrup, N. \& Nielsen, M. O. (2007). Estimation of fractional integration in the presence of data noise. Computational Statistics \& $\mathcal{E}$ Data Analysis, 51:3100-3114.

Hampel, F., Ronchetti, E., Rousseeuw, P., \& Stahel, W. (1986). Robust statistics: The approach based on influence functions. John Wiley \& sons.

Hurvich, C. \& Beltrão, K. (1990). Cross-validatory choice of a spectrum estimate and its connections with aic. Journal of Time Series Analysis, 11:121-137.

Hurvich, C. M., Deo, R., \& Brodsky, J. (1998). The mean square error of Geweke and Porter-Hudaks estimator of the memory parameter of a long-memory time series. Journal of Time Series Analysis, 19(1):19-46.

Ledolter, J. (1989). The effect of additive outliers on the forecast from arma models. International Journal of Forecasting, 5:231-240.

Lévy-Leduc, C., Boistard, H., Moulines, E., Taqqu, M., \& Reisen, V. A. (2011a). Asymptotic properties of U-processes under long-range dependence. The annals of statistics, 39:1399-1426.

Lévy-Leduc, C., Boistard, H., Moulines, E., Taqqu, M., \& Reisen, V. A. (2011b). Robust estimation of the scale and the autocovariance function of gaussian short and long-range dependent processes. Journal of Time Series Analysis, 32:135156 .

Ma, Y. \& Genton, M. (2000). Highly robust estimation of the autocovariance function. Journal of Time Series Analysis, 21:663-684.

Martin, D. \& Yohai, V. (1986). Influence functionals for time series. Annals of statistics, 14:781-818.

Martin, R. D. \& Thomson, D. (1982). Robust-resistant spectrum estimation. Procedings of the IEEE, 70(9):1097-1115.

Priestley, M. B. (1981). Spectral Analysis and Time Series. Academic Press. 
Reisen, V. A. (1994). Estimation of the fractional difference parameter in the $\operatorname{ARIMA}(p, d, q)$ model using the smoothed periodogram. Journal of Time Series Analysis, 15:335-350.

Rousseeuw, P. J. \& Croux, C. (1993). Alternatives to the median absolute deviation. Journal of the American Statistical Association, 88:1273-1283.

Spangl, B. \& Dutter, R. (2005). On robust estimation of power spectra. Austrian Journal of Statistics, 34(2):199-210.

Tatum, L. \& Hurvich, C. (1993). High breakdown methods of time series analysis. Journal of the Royal Statistical Society B, 55:881-896. 


\section{A. Appendix}

Lemma 1. Let $\left\{z_{t}\right\}$ be a contaminated process, with representation given by Eq. 4. Then

$$
f_{z}(\lambda)=f_{y}(\lambda)+\frac{1}{2 \pi} \gamma(1-\gamma) \sigma_{v}^{2} .
$$

Proof of Lemma 1. Following the definition given in Eq. 1 the spectrum of the contaminated process is such that

$$
f_{z}(\lambda)=\frac{1}{2 \pi}\left[R_{z}(0)+2 \sum_{h=1}^{\infty} R_{z}(h) e^{-i h \lambda}\right],
$$

where $R_{z}(0)=R_{y}(0)+\gamma(1-\gamma) \sigma_{v}^{2}$. Therefore,

$$
\begin{aligned}
f_{z}(\lambda) & =\frac{1}{2 \pi}\left[R_{y}(0)+2 \sum_{h=1}^{\infty} R_{y}(h) e^{-i h \lambda}\right]+\frac{1}{2 \pi} \gamma(1-\gamma) \sigma_{v^{*}}^{2} \\
& =f_{y}(\lambda)+\frac{1}{2 \pi} \gamma(1-\gamma) \sigma_{v}^{2} .
\end{aligned}
$$

Proof of Theorem 1. The proof is similar to that of Theorem 1 in Deo and Hurvich (2001), considering the result obtained from Lemma 1. 